\title{
COMPARATIVE STUDY OF METFORMIN AND VILDAGLIPTIN FOR MANAGEMENT OF PATIENTS WITH TYPE 2 DIABETES MELLITUS
}

\author{
Lakshman Das ${ }^{1}$, Mukut Roy², Ranjib Ghosh ${ }^{3}$
}

${ }_{1}^{1}$ Associate Professor, Department of Pharmacology, Tripura Medical College \& Dr. BRAM Teaching Hospital, Agartala, Tripura. ${ }^{2}$ Associate Professor, Department of Medicine, Tripura Medical College and Dr. BRAM Teaching Hospital, Hapania, Agartala, Tripura. ${ }^{3}$ Professor, Department of Pharmacology, Tripura Medical College and Dr. BRAM Teaching Hospital, Hapania, Agartala, Tripura.

\section{ABSTRACT}

\section{BACKGROUND}

Diabetes mellitus is a major health problem which increases the rate of morbidity and mortality. Cheap Metformin and costlier vildagliptin are the frequently used oral hypoglycaemic agents. The comparative effectiveness of metformin and vildagliptin has not been studied earlier. So, this study was conducted to compare the antidiabetic efficacy of metformin and vildagliptin and their effects on renal function in patients with type 2 diabetes mellitus.

\section{MATERIALS AND METHODS}

It was a prospective, open-labelled, non-randomised, phase IV clinical trial which was carried out over the duration of 12 weeks. The patients attending the medicine outpatient department of Tripura Medical College (TMC) hospital were enrolled in the study. Patients, who fulfilled the selection criteria, were allocated in two treatment groups. Group A was treated with metformin (Sustained release preparation) $500 \mathrm{mg}$ once daily and group B was treated with vildagliptin $50 \mathrm{mg}$ once daily. Measurement of body weight, fasting blood glucose (FPG), postprandial blood glucose (PPG), glycated haemoglobin ( $\mathrm{Hb}_{1 \mathrm{c}}$ ), serum urea, creatinine and urine albumin/creatinine ratio was performed at the initial visit and at the end of 12 weeks of treatment.

\section{RESULTS}

Metformin and vildagliptin significantly reduced the levels of FPG, PPG \& $\mathrm{HbA}_{1 \mathrm{c}}$ at week \#12 from their respective baseline values. There was similar reduction in the level of $\mathrm{HbA}_{1 \mathrm{c}}$ in both groups. Vildagliptin significantly reduced urine ACR at week \#12 from baseline value.

\section{CONCLUSION}

The study shows that metformin and vildagliptin have similar effect on glycaemic control, but vildagliptin exerts better renoprotective effect and there were no reports of serious adverse events.

\section{KEYWORDS}

Diabetes Mellitus, Metformin, Vildagliptin.

HOW TO CITE THIS ARTICLE: Das L, Roy M, Ghosh R. Comparative study of metformin and vildagliptin for management of patients with type 2 diabetes mellitus. J. Evolution Med. Dent. Sci. 2017;6(75):5372-5375, DOI: 10.14260/Jemds/2017/1165

\section{BACKGROUND}

Diabetes mellitus (DM) is a chronic disorder emerging as major health problem which increases the rate of morbidity and mortality. ${ }^{1}$ At present over 415 million people are living with diabetes across the globe, by 2040 this will rise to 642 million. ${ }^{2}$ A survey on Indian population showed that $4 \%$ of the adults suffered from DM in the year 2000 and it is expected to rise to $6 \%$ by the year $2025 .^{3}$

Nephropathy has been reported to occur in $20-40 \%$ of patients with diabetes and is the single leading cause of end stage renal disease, accounting for $>40 \%$ of patients requiring renal replacement therapy each year in developed countries. ${ }^{4}$ There are nearly $30 \%$ of chronic renal failure patients in India because of diabetic nephropathy. ${ }^{5}$

Financial or Other, Competing Interest: None.

Submission 18-07-2017, Peer Review 06-09-2017,

Acceptance 12-09-2017, Published 18-09-2017.

Corresponding Author:

Dr. Mukut Roy,

Associate Professor,

Department of Medicine,

Tripura Medical College and

Dr. BRAM Teaching Hospital,

Hapania, Agartala, Tripura.

E-mail:mukutdoc@gmail.com

DOI: $10.14260 /$ jemds $/ 2017 / 1165$
The major epidemiological studies support that glycosylated haemoglobin $\left(\mathrm{HbA}_{1 \mathrm{c}}\right)$ levels should be maintained as close to normal which may give longterm beneficial effects on the risk of diabetes complications. ${ }^{6}$

The cost of antidiabetic drugs is the major deciding factor for the patients' compliance. There exists a wide range of variation in the prices of drugs marketed in India and other countries of the world. ${ }^{7}$ Medical bankruptcy is a common phenomenon in India, as in other resource-challenged countries, where patients have to pay from pocket.

Metformin and vildagliptin are commonly used oral hypoglycaemic agents (OHAs). The comparative effectiveness of cheap OHA metformin and costlier OHA vildagliptin has not been studied earlier. ${ }^{8}$

So, the present study was conducted to evaluate the effectiveness of metformin with vildagliptin on glycaemic control and renal function in type $2 \mathrm{DM}$ patients.

\section{MATERIALS AND METHODS Objectives/Primary}

To compare the antidiabetic efficacy of metformin and vildagliptin in type- 2 diabetes mellitus. 


\section{Secondary}

1. To analyse the adverse drug reactions (ADRs) of metformin and vildagliptin.

2. To compare the effects of metformin and vildagliptin on renal function in the study subjects, if any.

A prospective, open-labelled, non-randomised, phase IV clinical trial was carried out over the duration of 12 weeks. The patients attending the medicine outpatient department of TMC hospital were enrolled in the study after explaining the aim of the study. Written informed consent was obtained from each patient. Prior approval of Institutional ethics committee was obtained [Permission Ref No.F.3 (PO75)/Inst. Ethical Com./SFTMC/2010-11/123284-123301 dated 05/08/2016].

\section{Inclusion Criteria}

- $\quad$ Adults of either gender aged between 18 to 60 years.

- Newly diagnosed type-2 diabetes patients with FPG $<200$ $\mathrm{mg} / \mathrm{dL}, \mathrm{PPG}<300 \mathrm{mg} / \mathrm{dL}$ and $\mathrm{HbA}_{1 \mathrm{c}}$ level from 6.5 to 10 .

- Patients with normal renal function.

\section{Exclusion Criteria}

- Patients with any complication of diabetes mellitus like retinopathy, neuropathy or nephropathy, etc.

- Patients with impaired liver function tests.

- Pregnant or lactating women.

- Patients taking medications that could affect blood glucose level, i.e. patients on non-selective $\beta$-blockers, diuretics and corticosteroids.

- Any contraindication to metformin/vildagliptin.

- $\quad$ Patients with known psychiatric illness.

Patients, who fulfilled the selection criteria, were allocated in two treatment groups (Group A \& Group B) by nonrandomised manner. In both groups, age and sex matched study subjects were selected. The decision to prescribe metformin or vildagliptin was made by the treating physician according to his/her normal medical practice, and patients were enrolled in the study only after the treatment decision had been made. Group A was treated with metformin (sustained release preparation) $500 \mathrm{mg}$ once daily and group B was treated with vildagliptin $50 \mathrm{mg}$ once daily.

Measurement of body weight, fasting blood glucose (FPG), postprandial blood glucose (PPG), $\mathrm{HbA}_{1 \mathrm{c}}$, serum urea \& creatinine and urine albumin/creatinine ratio (ACR) was performed at initial visit and at the end of 12 weeks of treatment. Patients were monitored continuously throughout the study for any adverse event (AE). Adverse drug reaction form of Pharmacovigilance Programme of India (PvPI) was filled up in case of any AE. World Health OrganizationUppsala Monitoring Centre (WHO-UMC) criteria $^{9}$ was used for causality assessment of AE.

\section{Sample Size}

Based on an effect size of 0.5, standard deviation of 0.7 , significance level $\alpha$ of 0.05 and power of the study as $80 \%$ power, the target number of evaluable subjects was 30 per group.

\section{Outcome}

Overall assessment was done at the end of the study period. The assessment of metformin/vildagliptin antidiabetic efficacy was done as follows-

1. Three-point scale $\mathrm{HbA}_{1 \mathrm{c}}$ assessment-

a. Excellent control: $\mathrm{HbA}_{1 \mathrm{c}}<6.5$

b. Good control: $\mathrm{HbA}_{1 \mathrm{c}}=6.5$ to 8

c. Poor control: $\mathrm{HbA}_{1 \mathrm{c}}>8$

2. Blood glucose levels-FPG \& PPG.

The Role of Metformin and Vildagliptin on Renal Function was assessed at the End of the Study by the following Parameters

1. Serum urea.

2. Serum creatinine.

3. Urine albumin-creatinine ratio.

\section{Statistical Analysis}

The data were analysed by SPSS version 17 . P value of $<0.05$ was considered significant. For intragroup comparison paired $t$ test and for intergroup comparison unpaired $t$ test were used. The data collected at 0 week will be used as the baseline against which changes during therapy will be compared. Chisquare test will be used to compare adverse effects between the groups. All statistical tests were two sided, and the results were presented as mean \pm standard deviation.

\section{RESULTS}

Out of 84 patients screened, 74 were enrolled for the study. Of the 74 patients, 39 (52.7\%) were male and 35 (47.3\%) were female. The patients were divided into two groups (group A \& group B) consisting 37 patients in each group. Out of 74 patients, 62 completed the study. Out of 12 patients who did not complete the study, 5 patients were lost during followup period and 7 patients discontinued treatment due to AEs (Figure-1). The mean age of the patients was 51 and 49 years in the groups A and B respectively.

There was no statistical difference in the baseline FPG, PPG, $\mathrm{HbA}_{1 \mathrm{c}}$, serum urea, serum creatinine, urine ACR and body weight between two groups.

\section{Hypoglycaemic Effect of Metformin and Vildagliptin}

Metformin \& vildagliptin significantly reduced $(\mathrm{p}<0.0001)$ the levels of FPG, PPG \& $\mathrm{HbA}_{1 \mathrm{c}}$ at week \#12 from their respective baseline values. In both groups there was good glycaemic control $\left(\mathrm{HbA}_{1 \mathrm{c}}=6.5-8\right)$ at the end of the study. The reduction in blood glucose level by vildagliptin was statistically significant $(p<0.001)$ as compared to metformin. But there was no significant change in the level of $\mathrm{HbA}_{1 \mathrm{c}}$ between the groups. (Table-1)

\section{Role of Metformin and Vildagliptin on Renal Function}

There was significant decrease $(\mathrm{p}<0.05)$ in serum urea level in vildagliptin group as compared to baseline value, but there was no such significant change in metformin group. In both the groups there was no significant change in serum creatinine levels at the end of 12 weeks as compared to baseline values. Vildagliptin significantly reduced urine ACR at week \#12 from baseline value $(\mathrm{p}<0.05)$, but in metformin group there was no significant change of urine ACR after 12 weeks of therapy. (Table-1). 


\section{Role of Metformin and Vildagliptin on Body Weight}

Metformin significantly reduced body weight $(\sim 3.31 \mathrm{~kg})$ at week \#12 as compare to baseline value $(\mathrm{p}<0.001)$, but in vildagliptin group there was no change in the body weight. (Table-1)

\section{Safety Analysis of Drug Therapy}

As per modified intention to treat (ITT), safety analysis of both metformin and vildagliptin was carried out. All patients who were receiving treatments were considered for safety analysis. Total seventeen patients developed AEs. The reported AEs were nausea, vomiting, abdominal bloating, epigastric pain, anorexia \& dizziness. Ten Patients in group A and seven patients in group B reported AEs. There were no significant difference of AEs between the groups (Chi square value $=0.6873, \mathrm{p}=0.407)$. These AEs were mild in nature. These lasted for about 2 weeks. Four (10.81\%) patients in group A and three (8.11\%) patients in group B discontinued the study due to AEs. Causality assessment showed that they were in the "possible" category.

\begin{tabular}{|c|c|c|c|c|}
\hline \multirow{2}{*}{} & \multicolumn{2}{|c|}{ Baseline Value } & \multicolumn{2}{c|}{ Value at the end of 12th Week } \\
\cline { 2 - 5 } & Metformin Group & Vildagliptin Group & Metformin Group & Vildagliptin Group \\
\hline FPG (mg/dL) & $171.31 \pm 7.37$ & $169.86 \pm 9.02$ & $125.47 \pm 5.89^{*}$ & $117.93 \pm 10.32^{*} \Phi$ \\
\hline PPG (mg/dL) & $225.7 \pm 6.71$ & $228.03 \pm 6.49$ & $171.75 \pm 8.78^{*}$ & $159.8 \pm 13.28^{*} \Phi$ \\
\hline $\mathrm{HbA}_{1 \mathrm{c}}(\%)$ & $8.27 \pm 0.39$ & $8.1 \pm 0.33$ & $6.98 \pm 0.1^{*}$ & $6.96 \pm 0.15^{*}$ \\
\hline Serum urea(mg/dL) & $32.33 \pm 5.68$ & $33.07 \pm 8.72$ & $31.98 \pm 5.03$ & $28 \pm 5.92^{\Delta}$ \\
\hline Serum Creatinine (mg/dL) & $0.66 \pm 0.18$ & $0.8 \pm 0.1$ & $0.64 \pm 0.12$ & $0.77 \pm 0.06$ \\
\hline Urine ACR (mg/g) & $18.66 \pm 3.06$ & $22 \pm 5.69$ & $18.22 \pm 1.52$ & $17.53 \pm 3.41^{* *}$ \\
\hline Body weight (kg) & $68.31 \pm 4.97$ & $66.33 \pm 4.61$ & $65 \pm 3.61^{\Psi}$ & $66.33 \pm 4.30$ \\
\hline \multicolumn{2}{|r|}{ Table 1. Effect of Metformin and Vildagliptin on Glycaemic Parameters, Kidney Function and Body Weight } \\
\hline
\end{tabular}

Values are expressed in mean standard \pm deviation.

$* p<0.0001$ as compare to baseline value.

${ }^{* *} \mathrm{p}=0.0003$ as compare to baseline value.

$\Delta \mathrm{p}=0.0156$ as compare to baseline value.

$\Psi \mathrm{p}=0.0076$ as compare to baseline value.

$\Phi p<0.001$ as compare to metformin group at the end of $12^{\text {th }}$ week.

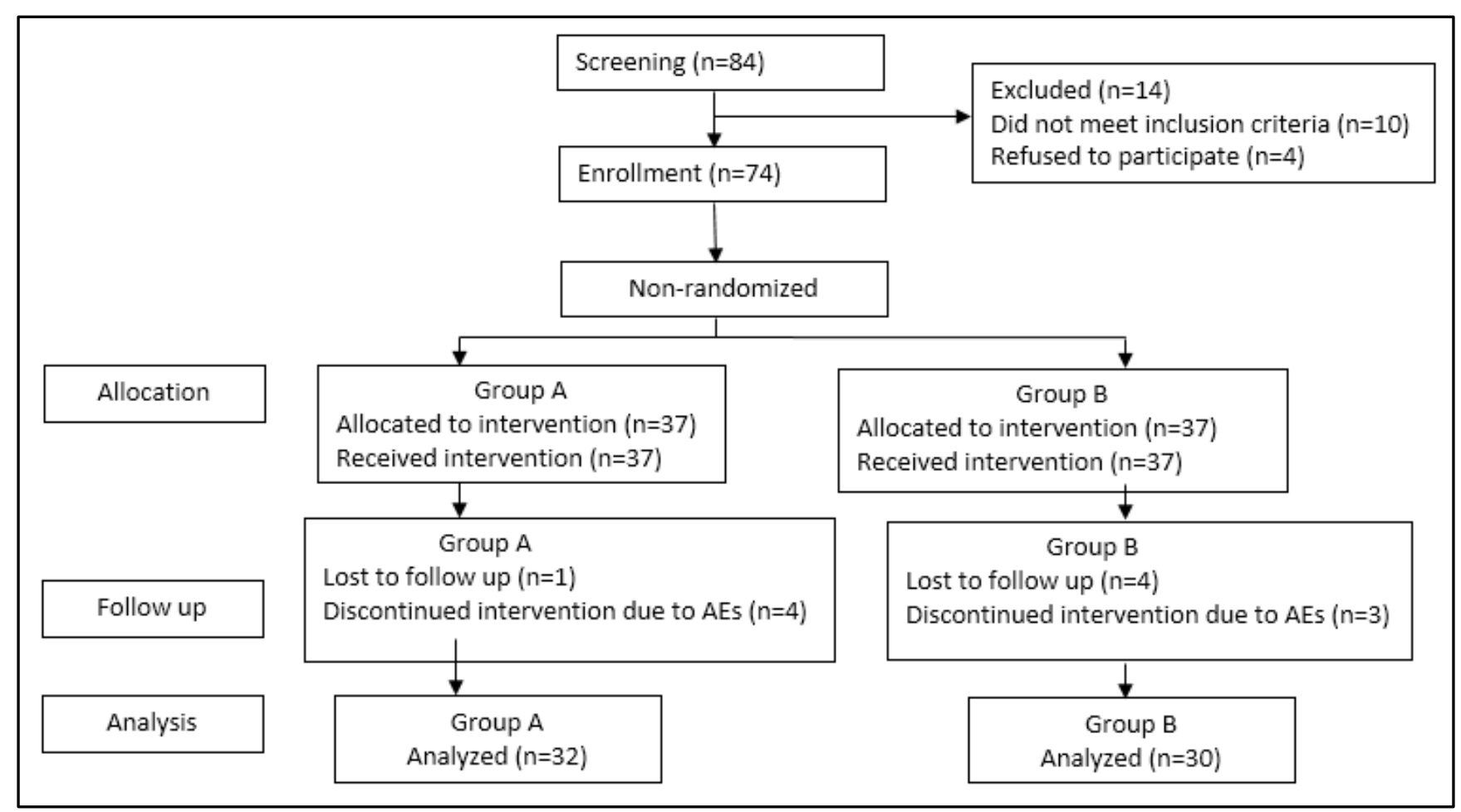

\section{DISCUSSION}

In this study, metformin significantly reduced the levels of FPG, PPG \& $\mathrm{HbA}_{1 \mathrm{c}}$ after 12 weeks of therapy. These findings are similar to that of ADAM CR et al. ${ }^{10}$ Metformin improves hyperglycaemia primarily by suppression of hepatic gluconeogenesis ${ }^{11}$ In addition to suppressing hepatic gluconeogenesis, metformin increases insulin sensitivity, enhances peripheral glucose uptake and increases fatty acid oxidation. ${ }^{12}$ Vildagliptin also significantly reduced the levels of FPG, PPG \& $\mathrm{HbA}_{1 \mathrm{c}}$ after 12 weeks of therapy. It acts by increasing the levels of GLP-1 and GIP by inhibiting DPP IV enzyme which is responsible for degradation of GLP-1 and GIP.13 Vildagliptin caused more reduction of FPG \& PPG but similar reduction of $\mathrm{HbA}_{1 \mathrm{c}}$ after 12 weeks of therapy as compared to that of metformin. 
Both metformin and vildagliptin protected renal function. Vildagliptin in addition improves kidney function as there was improvement in serum urea and urinary albumin excretion. Liu et $\mathrm{al}^{14}$ reported that vildagliptin attenuates kidney injury in streptozotocin-induced diabetic rats. In this same model, vildagliptin also significantly decreased proteinuria, albuminuria \& urinary ACR.

Metformin significantly reduced body weight $(\sim 3.31 \mathrm{~kg})$ which is comparable with the finding of Jack A. Yanovski et al15 But Overall weight neutrality has been seen with vildagliptin which is similar with findings of other studies. ${ }^{16,17,18,19}$ The overall weight neutrality seen with vildagliptin appears to be a class effect because the DPP-4 inhibitors, saxagliptin and sitagliptin have also been shown to produce improvements in glycaemic control, both as monotherapy and as add-on therapy to other oral agents, without significant change in body weight in most clinical trials. ${ }^{20,21}$

Safety analysis showed that there were no serious AEs, although $10.81 \%$ patients in group A and $8.11 \%$ patients in group B discontinued treatment due to AEs.

\section{CONCLUSION}

The study shows that metformin and vildagliptin have similar effect on glycaemic control, but vildagliptin exerts better renoprotective effect and there were no reports of serious adverse events.

\section{REFERENCES}

[1] Trplitt LC, Reasner AC, Isley LW, et al. Diabetes mellitus. In: Dipiro JT, Talbert RC, Matzke GR, et al. eds. Pharmacotherapy a pathophysiologic approach. 7th edn. New York: McGraw-Hill 2005:1333-67.

[2] Diabetes Atlas. $7^{\text {th }}$ edn. International Diabetes Federation, 2017. http://www.diabetesatlas.org/

[3] Day C. The rising tide of type 2 diabetes. $\mathrm{Br}$ J Diabetes Vasc Dis 2001;1(1):37.

[4] Melendez-Ramirez LY, Richards RJ, Cefalu WT. Complications of Type 1 Diabetes. Endocrinol Metab Clin N Am 2010;39(3):625-40.

[5] Vishwanathan V. Prevention of diabetic nephropathy: a diabetologist's perspective. Indian J Nephrol 2004;14:157-62.

[6] Colagiuri S. Optimal management of type 2 diabetes: the evidence. Diabetes Obes Metab 2012;14(Suppl 1):3-8.

[7] http://www.jbclinpharm.org December 30, 2014.

[8] Xu K, Evans DB, Kawabata K, et al. Household catastrophic health expenditure: a multicountry analysis. Lancet 2003;362(9378):111-7.

[9] Edwards IR, Aronson JK. Adverse drug reactions: definitions, diagnosis and management. Lancet 2000;356(9237):1255-9.
[10] Robinson AC, Burke J, Robinson S, et al. The effects of metformin on glycemic control and serum lipids in insulin-treated NIDDM patients with suboptimal metabolic control. Diabetes Care 1998;21(5):701-5.

[11] Kirpichnikov D, McFarlane SI, Sowers JR. Metformin: an update. Ann Intern Med 2002;137(1):25-33.

[12] Collier CA, Bruce CR, Smith AC, et al. Metformin counters the insulin-induced suppression of fatty acid oxidation and stimulation of triacylglycerol storage in rodent skeletal muscle. Am J Physiol Endocrinol Metab 2006;291(1):E182-E9.

[13] Sebokova E, Christ AD, Boehringer M, et al. Dipeptidyl peptidase IV inhibitors: the next generation of new promising therapies for the management of type 2 diabetes. Current Topics in Medicinal Chem 2007;7(6):547-55.

[14] Liu WJ, Xie SH, Liu YN, et al. Dipeptidyl peptidase IV inhibitor attenuates kidney injury in streptozotocininduced diabetic rats. J Pharmacol Exp Ther 2012;340(2):248-55.

[15] Yanovski JA, Krakoff J, Salaita CG, et al. Effects of metformin on body weight and body composition in obese insulin-resistant children. Diabetes 2011;60(2):477-85.

[16] Nathan DM, Buse JB, Davidson MB, et al. Medical management of hyperglycemia in type 2 diabetes: a consensus algorithm for the initiation and adjustment of therapy: a consensus statement of the American Diabetes Association and the European Association for the Study of Diabetes. Diabetes Care 2009;32(1):193203.

[17] Pi-Sunyer FX, Schweizer A, Mills D, et al. Efficacy and tolerability of vildagliptin monotherapy in drug-naïve patients with type 2 diabetes. Diabetes Res Clin Pract 2007;76(1):132-8.

[18] Dejager S, Razac S, Foley JE, et al. Vildagliptin in drugnaïve patients with type 2 diabetes: a 24-week, double-blind, randomized, placebo-controlled, multiple-dose study. Horm Metab Res 2007;39(3):218-23.

[19] Garber AJ, Foley JE, Banerji MA, et al. Effects of vildagliptin on glucose control in patients with type 2 diabetes inadequately controlled with a sulphonylurea. Diabetes Obes Metab 2008;10(11):1047-56.

[20] Rosenstock J, Sankoh S, List JF. Glucose-lowering activity of the dipeptidyl peptidase-4 inhibitor saxagliptin in drug-naive patients with type 2 diabetes. Diabetes Obes Metab 2008;10(5):376-86.

[21] Aschner P, Kipnes MS, Lunceford JK, et al. Effect of the dipeptidyl peptidase-4 inhibitor sitagliptin as monotherapy on glycemic control in patients with type 2 diabetes. Diabetes Care 2006;29(12):2632-7. 